\title{
Estimation of Salivary Glucose, Calcium, Phosphorus, Alkaline Phosphatase, and Immunoglobulin A among Diabetic and Nondiabetic Children: A Case-Control Study
}

\author{
${ }^{1}$ Kalyani Uppu, ${ }^{2}$ Suzan Sahana, ${ }^{3}$ Ghanashyam P Madu, ${ }^{4}$ Aron AK Vasa, ${ }^{5}$ Sowjanya Nalluri, ${ }^{6}$ Kumar J Raghavendra
}

\begin{abstract}
Introduction: Saliva is vital for oral health and helps to maintain oral homeostasis. It may show qualitative and quantitative variations owing to any changes in the systemic health. Diabetes mellitus (DM) is a metabolic disease and the individuals may be at higher risk for oral health problems.

Objective: The study was aimed to estimate the levels of various salivary components among diabetic and nondiabetic children with similar caries status and also to analyze possible association between caries status and possible caries determinants in the saliva of diabetic children.

Materials and methods: A total of 70 children in the age group of 6 to 13 years with minimal dental caries (Decayed, Missing and Filled Teeth index (DMFT/dmft $>1$ and $<5)$ ) were selected. Group I comprised of type I diabetic children and on medication for diabetes and group II included healthy nondiabetic children. Salivary samples were collected from the participants by passive drool method and estimation of all salivary parameters was done using autoanalyzer.
\end{abstract}

Results: Statistical analyses were done using Student's t-test and results are presented as mean \pm standard deviation (SD). There was a highly significant difference in mean glucose value between diabetic and nondiabetic children. Levels of salivary calcium, phosphorus, and salivary immunoglobulin A (s-IgA) did not show any significant difference between the two groups. There was also a statistically significant difference in the alkaline phosphatase (AP) levels, which was found to be higher in diabetics.

Conclusion: An elevation in the levels of salivary glucose and $\mathrm{AP}$ was evident in diabetic children, which can be a risk marker for dental caries. There was no correlation in the levels of salivary calcium, phosphorus, and s-IgA levels among diabetic and healthy children.

Clinical significance: The salivary factors evaluated in the study may prove to be useful measures of caries experience in diabetic children.

${ }^{1}$ Postgraduate Student, ${ }^{2,4,5}$ Professor, ${ }^{3}$ Professor and Head ${ }^{6}$ Senior Lecturer

${ }^{1-6}$ Department of Pedodontics and Preventive Dentistry, Saint Joseph Dental College, Eluru, Andhra Pradesh, India

Corresponding Author: Suzan Sahana, Professor, Department of Pedodontics and Preventive Dentistry, Saint Joseph Dental College, Eluru, Andhra Pradesh, India, Phone: +919886231079 e-mail: drsuzansahana@gmail.com
Keywords: Alkaline phosphatase, Calcium, Nondiabetic healthy children, Phosphorus, Salivary glucose, s-IgA, Type I diabetic children.

How to cite this article: Uppu K, Sahana S, Madu GP Vasa AAK, Nalluri S, Raghavendra KJ. Estimation of Salivary Glucose, Calcium, Phosphorus, Alkaline Phosphatase, and Immunoglobulin A among Diabetic and Nondiabetic Children: A Case-Control Study. Int J Clin Pediatr Dent 2018;11(2):71-78.

Source of support: Nil

Conflict of interest: None

\section{INTRODUCTION}

Oral cavity is an extremely dynamic and unique environment, the only place in the body where mineralized tissues are exposed to external environment that involves complex interactions between different surfaces constantly exposed to saliva. To a large extent, saliva fosters oral health and no other etiological factor influences the outcome of dental caries as much as saliva does.

Dental caries is a multifactorial disease and the hallmark of this is demineralization which is initiated by acidogenic plaque flora and low salivary flow leading to slow clearance, poor buffering, and reduced supply of calcium to repair the altered dental tissues. The saliva, by constantly bathing the teeth and oral mucosa, functions as cleansing solution, a lubricant, a buffer, and an ion reservoir of calcium and phosphate, which are essential for remineralization of initial carious lesion. ${ }^{1}$

Low salivary buffering capacity, low calcium and phosphate levels show a pronounced link to increased caries. Maintenance of the equilibrium between demineralization and remineralization depends on the ionic concentration of calcium and phosphate in saliva, which in turn is influenced by AP levels. Variations in the levels of salivary enzymes like AP cause changes in phosphate levels, which lead to initiation and progression of dental caries. $^{2}$

Salivary constituents are affected in various local and systemic conditions. Among the various systemic diseases, DM is the fifth most common metabolic disorder routinely encountered in the world and its prevalence is constantly on the rise. ${ }^{3}$ According to the 6th edition 
of the International Diabetes Federation, diabetes atlas, India has 3 new cases of type I DM per 1 lakh children of 0 to 14 years. It is a complex multisystem disorder characterized by a relative or absolute insufficiency of insulin secretion and/or concomitant resistance to the metabolic action of insulin on target tissues. There is evidence that diabetic patients have saliva secretion and composition different from nondiabetic subjects. It has been suggested that hyperglycemia is associated with decreased salivary secretion and high salivary glucose levels, particularly in cases of severe insulin deficiency. Consequently, an increased cariogenic challenge in such individuals can be expected. ${ }^{4}$

Alterations in salivary amount and quality affect oral health. The alterations observed may be related to the levels of glucose, calcium, enzyme activity, and IgA as well. Normal glucose level in saliva is 0.5 to $1.00 \mathrm{mg} / 100 \mathrm{~mL}$, which does not significantly affect oral health or support the growth of microorganisms. However, higher salivary glucose levels favor the proliferation of microorganisms and enhance their colonization on teeth and oral mucous membrane. ${ }^{5}$ Furthermore, it is possible that increased concentration of IgA in saliva have protective role against caries development. $^{6}$

The salivary components in diabetic children too may suffer variations that can be detected by chemical determinations. Despite all the aforementioned risk factors for dental caries, the relationship between dental caries and diabetes remains controversial. ${ }^{6}$

However, most of the clinical trials estimating the salivary parameters are carried out in adults, which dictates the need to recognize significant association between the levels of salivary factors linked to dental caries in children with and without DM. Hence, the present study was aimed to estimate the levels of salivary glucose, calcium, phosphorus, AP, and $\operatorname{Ig} \mathrm{A}$ among diabetic and healthy

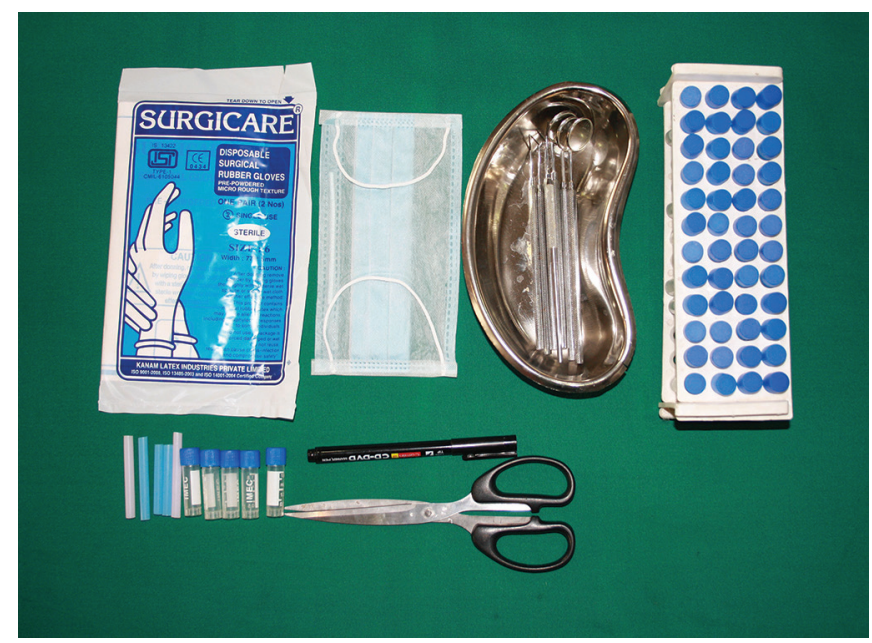

Fig. 1: Armamentarium used for the study nondiabetic children with caries and also to analyze the possible association between the caries status and possible caries determinants.

\section{MATERIALS AND METHODS}

This randomized clinical trial of diabetic and healthy children was conducted after obtaining clearance from the institutional ethical board. The study population was selected based on the duration of DM and hemoglobin A1c levels. To unify the cases, children affected with diabetes with a minimum duration of 2 years and also on medication for the same were selected.

The study comprised a total of 70 children inclusive of both sexes in the age group of 6 to 13 years. Both diabetic and healthy children who had minimal dental caries $(\mathrm{DMFT} / \mathrm{dmft}>1$ and $<5)$ were selected and grouped as follows:

- Group I: 35 children with type I DM and on medication for diabetes (study group).

- Group II: 35 healthy children (control group).

\section{Exclusion Criteria (Study Group)}

- Cases with acute oral afflictions, acute systemic infections, and chronic debilitating diseases.

- Patients with severe diabetic complications and other systemic illness or on medications other than those for diabetes.

\section{Selection of Individuals}

Informed consent was obtained from all the children/ parents for participation in the study.

The intraoral examination was performed to assess the DMFT/dmft in both normal and diabetic children (Figs 1 and 2). To unify the cases, children with minimal caries, i.e., DMFT $>1$ and $<5$, only were included in the

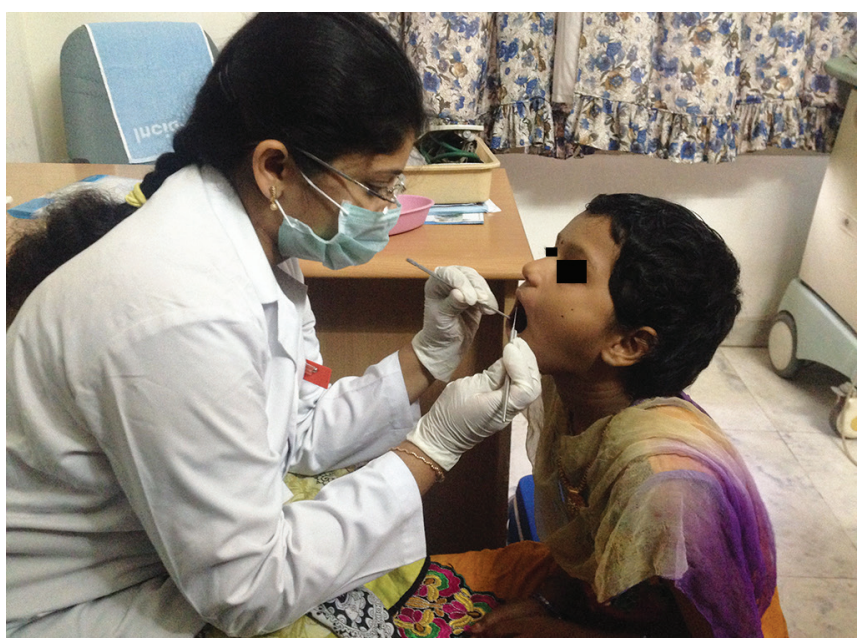

Fig. 2: Examination of participants 
study. Children who fulfilled the inclusion criteria were assigned for saliva collection.

The passive drool method was employed for the collection of whole saliva into a small vial.

\section{Participant Preparation}

Participants were instructed not to consume food (neither solids/liquids) 2 hours before and were asked to rinse their mouth with water 20 minutes prior to collection. The circadian rhythm can change the composition of saliva in the same individual at different times of the same day. To control the circadian variation, samples from all the children were collected between 10 am and 11.30 am. ${ }^{7}$ Each research participant was given a straw piece and a cryovial. They were instructed to allow saliva to pool in the mouth and with head tilted forward, saliva was made to drool down the straw and collect in the cryovial (Fig. 3) (It is normal for saliva to foam, so a vial with twice the capacity of the desired sample volume was used.) It was repeated as often as necessary until 2 mL was sufficiently collected. The samples were stored after collection at $\left(4^{\circ} \mathrm{C}\right)$ and were transported to the lab in a portable icebox (Fig. 4) within 24 hours.

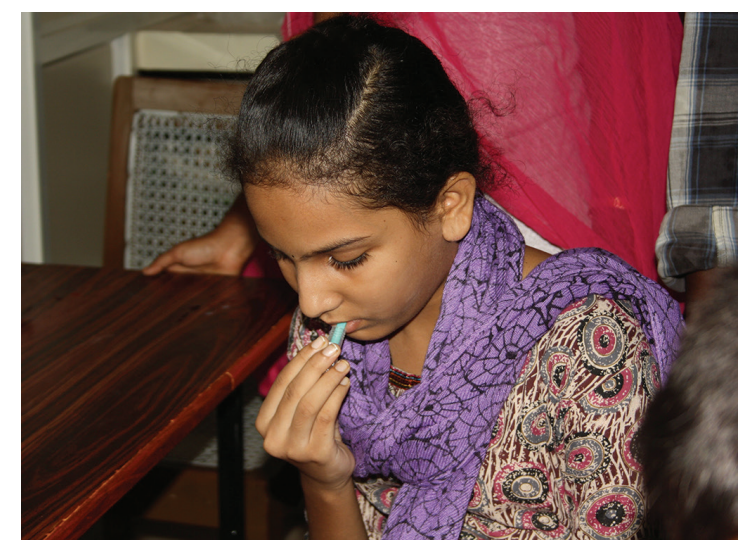

Fig. 3: Saliva collection (passive drool method)

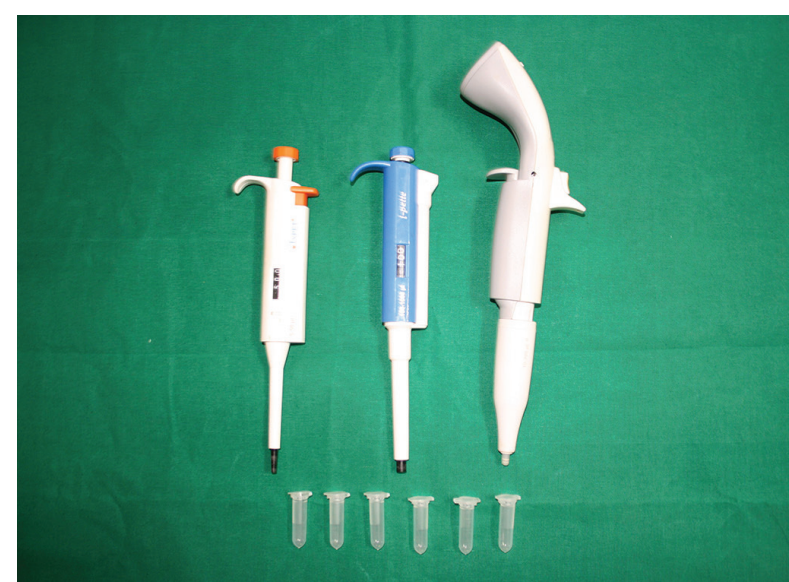

Fig. 5: Micropipettes for dilution of saliva
On the day samples were to be assayed, it was brought to room temperature, vortexed, and then centrifuged for 15 minutes at approximately 3,000 RPM $(1,500 \times \mathrm{g})$. Assays were performed using only clear saliva, avoiding any sediment present in the bottom of the tube (Fig. 5).

All the samples were subjected to analyses in a fully automated analyzer (Fig. 6) with biochemistry and ELISA reader (Chemwel plus, CPC diagnostics, Awareness technologies, USA), which works on the principle of atomic absorption spectrophotometer (Fig. 7).

- Salivary glucose estimation was performed using the glucose oxidase-peroxidase end-point method.

- Salivary calcium and phosphorus levels were estimated by colorimetric method (Fig. 8).

- For the estimation of AP, enzymatic assay kit was used that measures the concentration of AP using a direct, plate-based, colorimetric reaction.

- For the quantitative determination of s-IgA, immunoenzymatic colorimetric method was used. This s-IgA enzyme-linked immunosorbent assay (ELISA) test was based on simultaneous binding of human $\operatorname{Ig} \mathrm{A}$ to two antibodies, one monoclonal immobilized on a microwell plate, the other with polyclonal conjugated with horseradish peroxidase. The s-IgA concentration

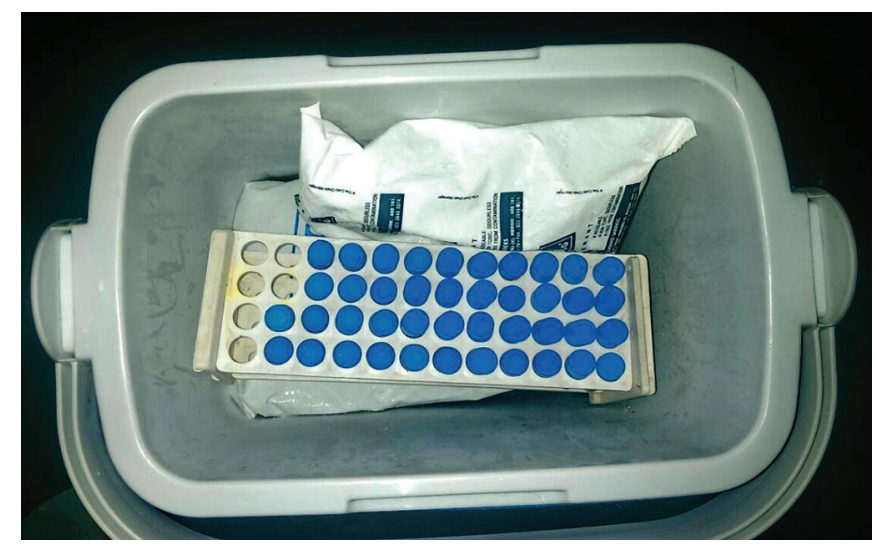

Fig. 4: Portable icebox for saliva storage

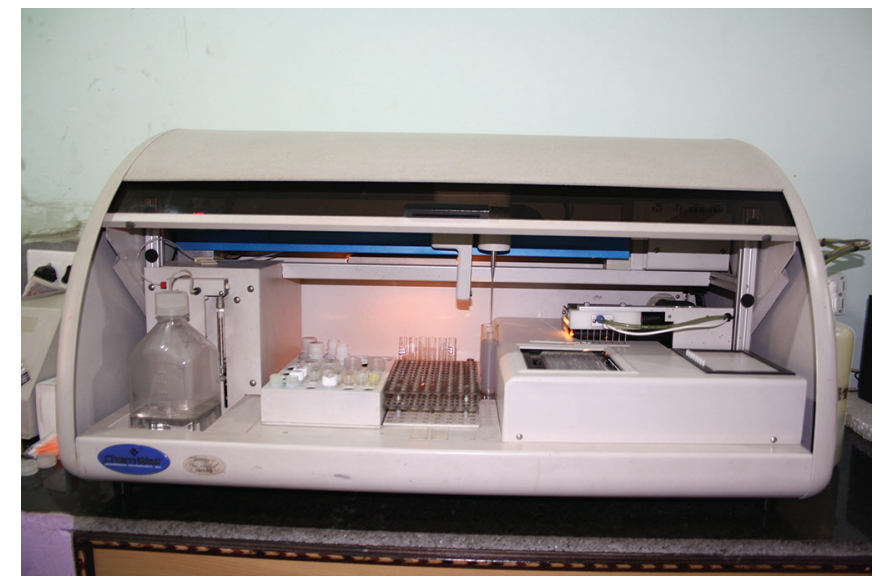

Fig. 6: Chemwell autoanalyzer (Awareness tech, USA) 


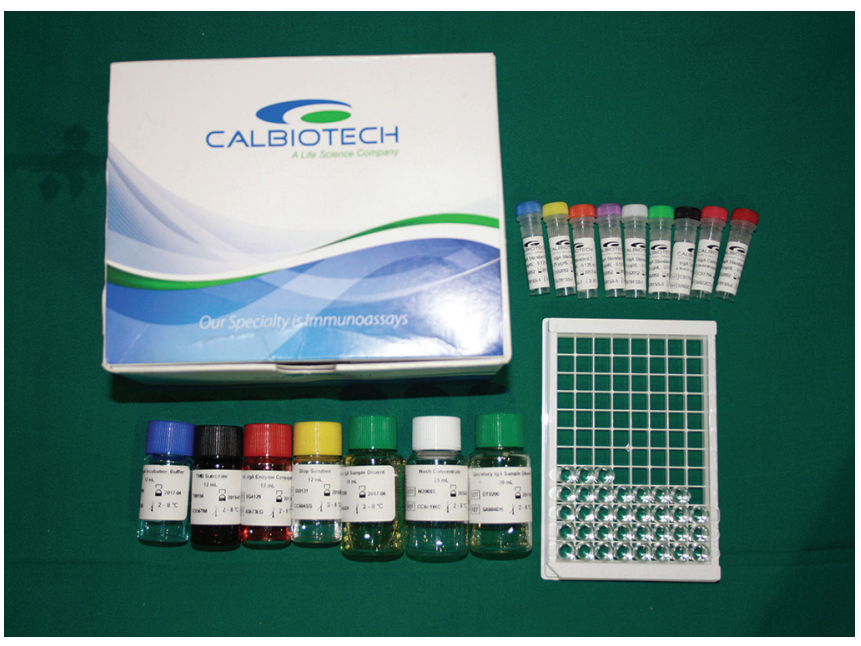

Fig. 7: Calbiotech ELISA kit

Table 1: Mean and SD of diabetic and nondiabetic children with respect to age and gender

\begin{tabular}{llll}
\hline \multicolumn{4}{c}{ Sample distribution } \\
\hline No of subjects & & Study & Control \\
Age (yrs) & Mean \pm SD & $10.1 \pm 2.3$ & 35 \\
& Range & $6-13$ & $8.2 \pm 1.1$ \\
Gender & Male & 13 & $6-13$ \\
& Female & 22 & 21 \\
& & & 14 \\
\hline
\end{tabular}

Table 3: Mean and SD of diabetic and nondiabetic children with respect to salivary calcium

\begin{tabular}{lllll}
\hline \multicolumn{5}{c}{ Calcium } \\
\hline Groups & Mean & SD & Min & Max \\
\hline Study & 2.66 & 1.75 & 0.00 & 8.10 \\
Control & 3.81 & 3.16 & 0.00 & 12.90 \\
Mean difference & 1.15 & & & \\
t-value & 1.91 & & & \\
p-value & 0.06, NS & & &
\end{tabular}

NS: Not significant

Table 5: Mean and SD of diabetic and nondiabetic children with respect to salivary AP

\begin{tabular}{lllll}
\hline \multicolumn{5}{c}{$A P$} \\
\hline Groups & Mean & SD & Min & Max \\
\hline Study & 22.09 & 11.07 & 0.00 & 48.50 \\
Control & 15.21 & 7.91 & 1.10 & 38.20 \\
Mean difference & 6.89 & & & \\
t-value & 2.98 & & & \\
p-value & $0.004, \mathrm{~S}$ & & & \\
\hline S: Significant & \multicolumn{5}{c}{}
\end{tabular}

S: Significant

in the sample was calculated based on a series of standard. The color intensity was proportional to the s-IgA concentration the sample.

\section{RESULTS}

Statistical analysis was performed by mean, SD, and Student's t-test. The range, mean scores, and SDs for diabetic

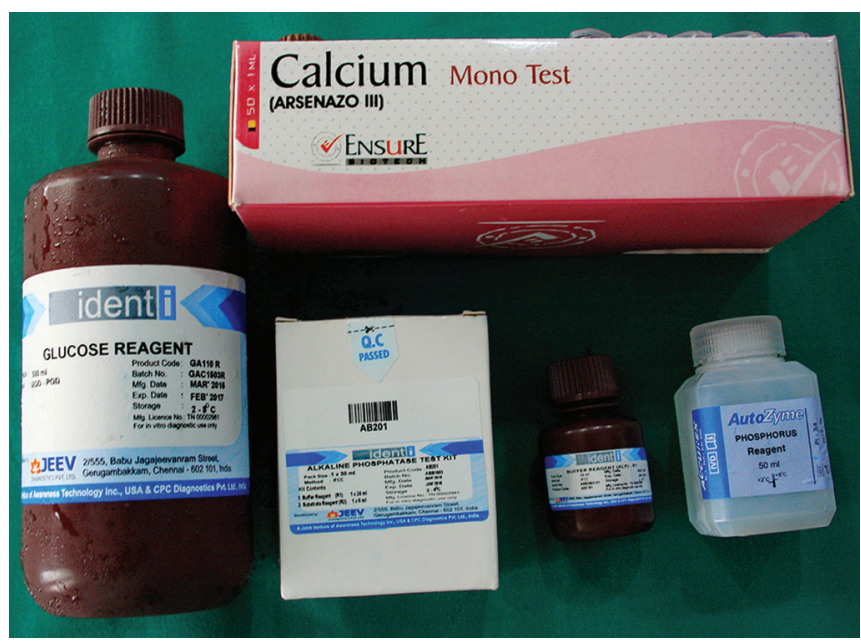

Fig. 8: Reagents used for the study

Table 2: Mean and SD of diabetic and nondiabetic children with respect to salivary glucose

\begin{tabular}{lllll}
\hline \multicolumn{5}{c}{ Glucose } \\
\hline Groups & Mean & SD & Min & Max \\
\hline Study & 4.51 & 2.83 & 1.00 & 12.00 \\
Control & 1.32 & 1.17 & 0.00 & 5.00 \\
Mean difference & 3.19 & & & \\
t-value & 6.09 & & & \\
p-value & $<0.001$, HS & & \\
\hline HS: Highly significant & & & &
\end{tabular}

Table 4: Mean and SD of diabetic and nondiabetic children with respect to salivary phosphorus

\begin{tabular}{lllll}
\hline \multicolumn{5}{c}{ Phosphorus } \\
\hline Groups & Mean & SD & Min & Max \\
\hline Study & 11.66 & 2.70 & 6.70 & 18.00 \\
Control & 11.77 & 2.30 & 1.10 & 38.20 \\
Mean difference & 0.11 & & & \\
t-value & 0.18 & & & \\
p-value & 0.86, NS & & & \\
\hline NS: Not significant & & &
\end{tabular}

Table 6: Mean and SD of diabetic and nondiabetic children with respect to s-lgA

\begin{tabular}{lllll}
\hline \multicolumn{5}{c}{ Salivary IgA } \\
\hline Groups & Mean & SD & Min & Max \\
\hline Study & 36.44 & 16.30 & 13.52 & 72.65 \\
Control & 34.26 & 12.82 & 12.39 & 56.53 \\
Mean difference & 2.18 & & & \\
t-value & 0.65 & & & \\
p-value & 0.52, NS & & \\
\hline NS: Not significant & \multicolumn{5}{l}{}
\end{tabular}

and control group are presented in Table 1. The mean values of salivary components estimated are exhibited in Tables 2 to 6.

In group I (diabetic children), the mean concentrations of salivary glucose, calcium, phosphorus, $\mathrm{AP}$, and s-IgA were $4.51 \mathrm{mg} / \mathrm{dL}, 2.66 \mathrm{mg} / \mathrm{dL}, 11.66 \mathrm{mg} / \mathrm{dL}, 22.09 \mathrm{U} / \mathrm{L}$, and $36.44 \mathrm{mg} / \mathrm{dL}$ respectively. 


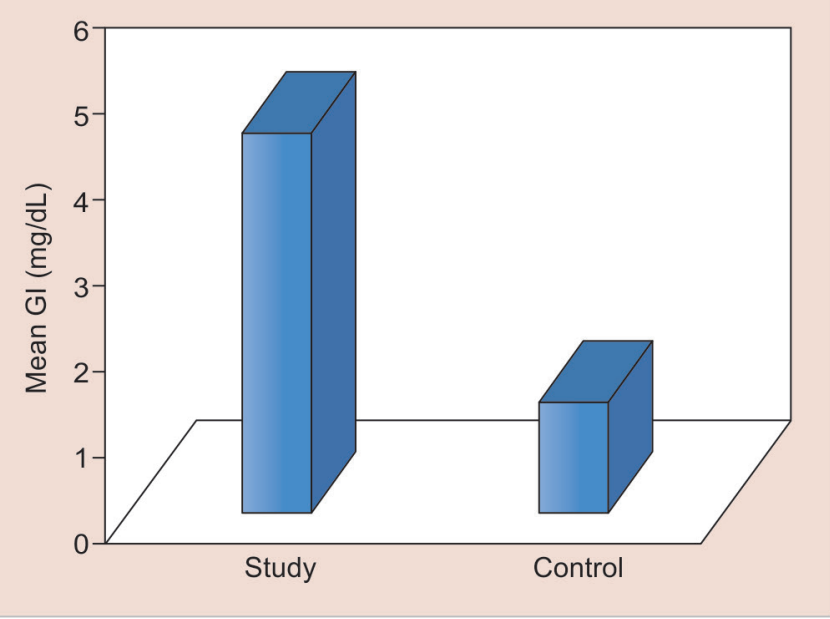

Graph 1: Mean salivary glucose levels in two groups

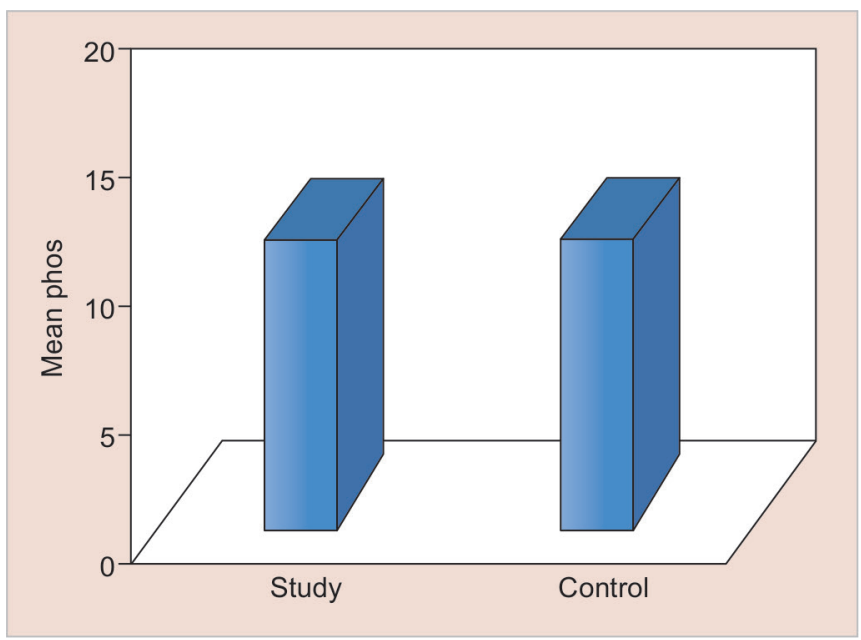

Graph 3: Mean salivary phosphate levels in two groups

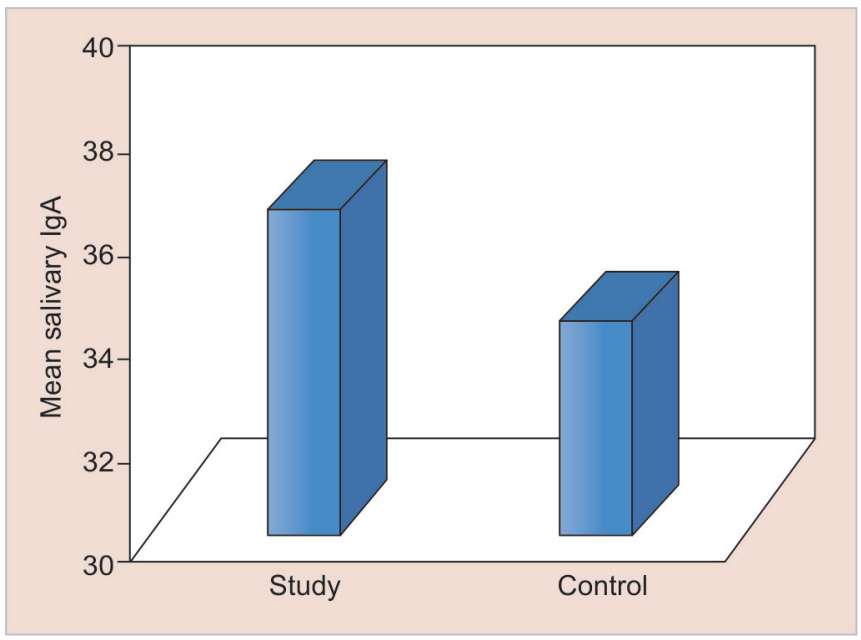

Graph 5: Mean s-IgA levels in two groups

In group II (nondiabetic children), the mean concentrations of salivary glucose, calcium, phosphorus, $\mathrm{AP}$, and s-IgA were $1.32 \mathrm{mg} / \mathrm{dL}, 3.81 \mathrm{mg} / \mathrm{dL}, 11.77 \mathrm{mg} / \mathrm{dL}, 15.21$ $\mathrm{U} / \mathrm{L}$, and $34.26 \mathrm{mg} / \mathrm{dL}$ respectively.

The values of comparison are depicted in Graphs 1 to 5 .

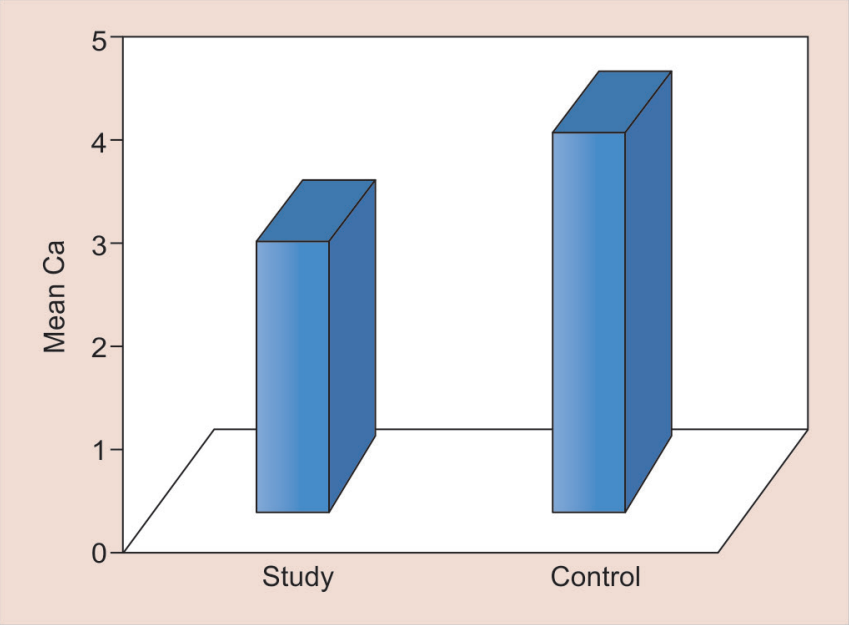

Graph 2: Mean salivary calcium levels in two groups

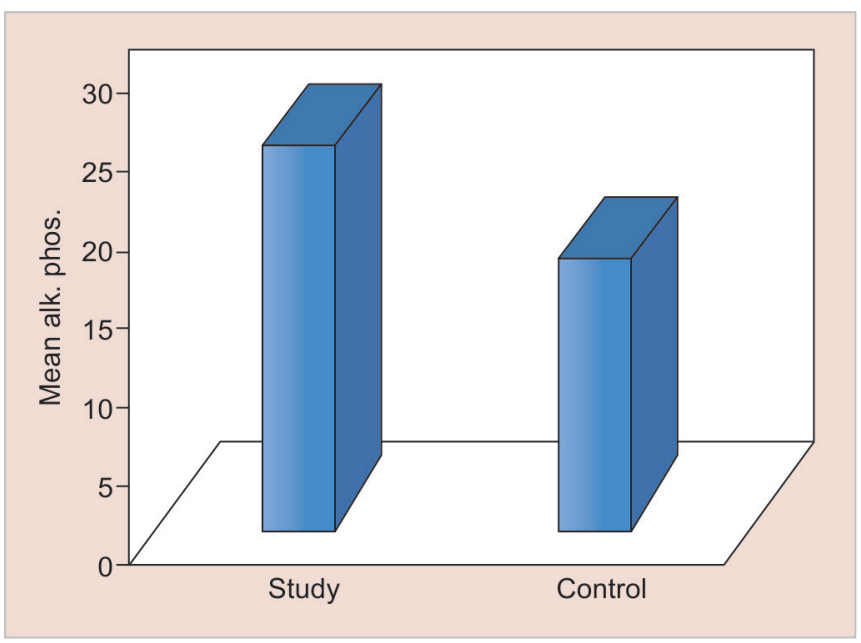

Graph 4: Mean salivary AP levels in two groups

\section{DISCUSSION}

One of the goals in dentistry today is the provision of regular care to high-risk groups of the population, among which are the medically compromised children, such as those suffering with type I DM. Continuous monitoring of oral health of these children leads to dramatic improvement of oral ecology and a decrease in the caries incidence.

The present study assessed the various salivary components like glucose, calcium, phosphorus, $\mathrm{AP}$, and s-IgA among diabetic children because these elements have a role in maintaining the equilibrium between the dissolution of enamel by acids produced by oral microbes and saliva of the oral cavity. The study was anticipated to estimate the probable association linked to salivary factors and the risk of systemic disease on child's oral health when compared with healthy children.

The present investigation included children in the age group of 6 to 13 years, as this is the developmental period of transitional dentition and the duration of overt diabetes may exert an influence on the mineralization of 
newly erupted teeth and also affect the inorganic constituents of saliva.

The results of the present study indicated significantly higher glucose levels among diabetics when compared with healthy children. These results are in agreement with the reports of previous studies by Panchbai et $\mathrm{al}^{5}$ and Abhikshyeet et al. ${ }^{9}$ Earlier reports have quoted various reasons for the elevated glucose content in the salivary secretion of diabetic individuals. Lopez et al stated that the salivary glands act as filters of blood glucose that would be altered by hormonal or neural regulation. Glucose also makes its way from the gingival crevicular fluid into the saliva. Even under pathologic conditions like infections and inflammations of salivary glands, raised glucose levels can be seen. ${ }^{8}$ Persistent hyperglycemia leads to microvascular changes in the blood vessels, as well as basement membrane alteration in the salivary glands. This leads to increased leakage of glucose from the ductal cells of salivary gland, thereby increasing the glucose content in saliva. ${ }^{9}$

Decreased secretion of saliva combined with an increased amount of glucose in the saliva and exudate from gingival crevices can presumably be a caries-promoting factor. ${ }^{10}$

Increased levels of glucose in the saliva stimulate bacterial growth and the production of lactic acid, leading to a decrease of the $\mathrm{pH}$ and buffering capacity of the saliva, and converts them to risk factors for the development of dental caries. Increased oral acidity resulting from the high level of glucose in the saliva causes a change in the dental biofilm and facilitates the colonization of Streptococcus mutans and Lactobacillus. ${ }^{11}$

Though numerous studies found higher salivary glucose levels in diabetic patients than in nondiabetics, Belazi et $\mathrm{al}_{,}{ }^{11}$ Bakianian Vaziri et $\mathrm{al}_{,}{ }^{12}$ Gheena et $\mathrm{al},{ }^{13}$ Shahbaz et al, ${ }^{14}$ and Kakoie et al ${ }^{15}$ did not report any difference with respect to salivary glucose levels in normal and diabetic individuals. The difference may be due to the diabetic status and better glycemic control of the studied sample. These differences in the analysis of results might also be due to different methods employed for the measurement of salivary glucose or even the method of collecting the samples. In addition, the level of maintaining oral hygiene and plaque on teeth can influence the salivary glucose levels.

According to Twetman et $\mathrm{al}_{,}{ }^{16}$ the cut-off value of stimulated resting salivary glucose concentration for poor metabolic control and caries-associated risk factor is $>12.5 \mathrm{mg} / \mathrm{L}$. However, the present investigation showed the mean glucose levels in diabetics to be well within the range, and hence, the associated risk might also be minimized.

\section{Salivary Calcium and Phosphorus}

In the present study, the mean calcium concentration in diabetic children with dental caries was $2.66 \pm 1.75$ and that of nondiabetic children was $3.81 \pm 3.16$, which is slightly high. Mean salivary phosphorus levels in diabetic children was found to be $11.66 \pm 2.70$ and that of nondiabetic children was $11.77 \pm 2.30$ with a mean difference of 0.11 $\mathrm{mg} / \mathrm{dL}$, which is not statistically significant $(\mathrm{p}>0.05)$. Hence, a nonsignificant difference was revealed between calcium and phosphorus levels among the diabetics and healthy children.

As reported by Damle et $\mathrm{al}_{1}{ }^{17}$ the levels of calcium, phosphorus, and AP are not significantly altered in cariesactive and caries-free persons. Lasisi and Fasanmade ${ }^{18}$ reported that calcium levels in diabetics are not significantly different from that of the nondiabetics. Individuals with increasing DMFT show a decrease in minerals like calcium and phosphorus in both serum and saliva. ${ }^{19}$

The adequate level of calcium, phosphate, and even fluoride is responsible for the significant deposition of these minerals in plaque that greatly reduces the development of caries in the adjacent enamel. This indicates that diabetic patients are more prone to develop dental caries if the calcium, phosphorus level decreases and the levels of AP increase. ${ }^{20}$ Since the values estimated among diabetics in the present study were on par with the healthy children with respect to salivary calcium and phosphorus, the susceptibility also may be considered to be minimum.

Factors to be taken into consideration here include newly diagnosed cases if any, among the sample population studied because diabetes of less than 2-year duration may hardly have any influence on dental development. ${ }^{21,22}$

\section{Alkaline Phosphatase}

Alkaline phosphatase is a nonspecific phosphomonoesterase that has different isoenzymes produced by different cell types, such as polymorphonuclear leukocytes, osteoblasts, macrophages, and fibroblast within alveolar bone and/or salivary glands. It has been shown in different studies that higher AP activity is related to periodontal disease and dental caries and it seems that the function of this protein is relatively dependent on salivary $\mathrm{pH}$ and buffering. ${ }^{23}$

Mean salivary AP levels in diabetic children (22.09 \pm 11.07) were much higher when compared with nondiabetic children $(15.21 \pm 7.91)$ with a mean difference of 6.89 $\mathrm{mg} / \mathrm{dL}$. A statistically significant difference $(\mathrm{p}<0.05)$ was observed in the present investigation between the two groups with respect to salivary AP levels.

Hegde et al, ${ }^{22}$ who reported AP activity in saliva to be higher in diabetic adults, substantiate the findings with the results of the present study. It is well established that initial caries lesion formation seen clinically as the white 
spot lesion is marked by a subsurface demineralization with an intact surface layer which can be remineralized, reestablishing the demineralization-remineralization equilibrium.

Studies documented by Vijayaprasad et $\mathrm{al}^{24}$ and Gandhy and Damle ${ }^{25}$ have shown a positive and direct correlation between salivary AP level and dental caries. The same authors in a different study reported that there was an increase in the levels of caries with an increase in the levels of AP levels in both serum and saliva. They reported calcium levels in serum and saliva to be decreased in caries-prone individuals, thus showing a significant correlation between serum and salivary AP and calcium levels.

Vijayaprasad et $\mathrm{al}^{24}$ and Bakas $^{20}$ also reported AP activity to be significantly higher in caries-prone groups, but observed no significant difference of calcium and phosphorus levels between caries-free, minimal caries, and caries-prone groups. The present study too did not reveal any significant difference between calcium and phosphorus levels between type I DM and nondiabetics. Although salivary AP can balance demineralization and remineralization processes of enamel, there is no evidence regarding its effects on the concentrations of calcium and phosphate in saliva.

In contrast to the present study, Jazaeri et $\mathrm{al}^{23}$ reported no significant relation between salivary AP activity and calcium and phosphate concentrations in saliva. It seemed that the other controlling mechanism was responsible for maintaining calcium and phosphate at a normal range.

\section{Salivary IgA}

Mean s-IgA level in diabetic children was $36.44 \pm 16.30$ and that of nondiabetic children was $34.26 \pm 12.82$. Though the levels of s-IgA were high among diabetic children, the difference was not statistically significant $(\mathrm{p}>0.05)$.

Salivary IgA antibodies help oral immunity by preventing microbial adherence, neutralizing enzymes, toxins, and viruses, or by acting in synergy with other factors, such as lysozyme and lactoferrin. Gandhy and Damle $^{25}$ reported that s-IgA levels in children with no caries were significantly higher, suggesting a possible protective role of IgA in the prevention of dental caries. It could be due to the deficient transport mechanism, stimulation of the immune system via pulp, deficient local immunoglobulin synthesis, and molecular size of IgA.

In the present study, on comparison of s-IgA levels, diabetic children had higher levels of s-IgA when compared with nondiabetic children. However, the difference was not statistically significant. This is in agreement with the results of Rashkova et $\mathrm{al}^{26}$ and Bakianian Vaziri et $\mathrm{al}^{12}$ who reported that IgA levels are not significantly altered in either type I DM or type II DM compared with healthy controls. Contrary to this, Harrison showed significantly higher levels of s-IgA in uncontrolled diabetic children only, whereas no difference between controlled DM and healthy children. ${ }^{27}$

Watanabe et $\mathrm{al}^{28}$ demonstrated a significant increase in s-IgA levels in diabetic patients. They suggested that it could be related to local factors, such as calculus and higher bacterial plaque accumulation in these patients. The findings of this study on s-IgA levels were in contrast with other studies; these differences may be due to differences in the type of saliva collected (stimulated or unstimulated), the saliva collection methods, the stage and the metabolic control status of the disease.

The average values of s-IgA in the saliva of children with diabetes are close to those of the healthy children in the present study. Due to the initial phase of diabetes development, the endocrine system does not seem to influence the local secretary oral immunity to a greater extent.

The present study showed nonsignificant values with respect to calcium and phosphorus. This is attributed to the fact that the systemic effects of diabetes might not have much influence on tooth mineralization and concentration of inorganic constituents of saliva. However, higher glucose levels among diabetics may be due to more permeability of salivary glucose. Levels of s-IgA too were not a significant factor among diabetics and hence protection against developing caries can be anticipated on par with the normal children.

\section{CLINICAL SIGNIFICANCE}

The salivary factors evaluated here may prove to be useful measures of caries determinants in diabetic children which may help pediatric dentists to combat the ill effects of diabetes on oral health and target preventive measures appropriately.

\section{CONCLUSION}

The following conclusions were drawn from the present study:

- Despite a difference in systemic health status, the diabetic children showed minimum variation in salivary composition and oral health.

- There was no correlation in the levels of salivary calcium, phosphorus, and s-IgA levels among diabetic and healthy children.

- An elevation in the levels of salivary glucose and AP was evident in diabetic children, which can be risk markers for dental caries. 
However, the clinical interpretation of results obtained in this case-control study should be further substantiated incorporating a larger sample size in order to conclude the specific role played by saliva as a host-protective factor in diabetic children.

\section{REFERENCES}

1. Iqbal S, Kazmi F, Asad S, Mumtaz M, Khan AA. Dental caries \& diabetes mellitus. Pak Oral Dent J 2011 Jun;31(1):58-61.

2. Kaur A, Kwatra KS, Kamboj P. Evaluation of non microbial salivary caries activity parameters and salivary biochemical indicators in predicting dental caries. J Indian Soc Pedod Prev Dent 2012 Jul-Sep;30(3):212-217.

3. Nagalaxmi V, Priyanka V. Can saliva be a marker for predicting type 1 diabetes mellitus? A pilot study. J Indian Acad Oral Med Radiol 2011 Oct-Dec;23(4):579-582.

4. Das AK. Type 1 diabetes in India: overall insights. Indian J Endocr Metab 2015 Apr;19(Suppl 1):S31-S33.

5. Panchbai SA, Degweker SS, Bowte RR. Estimation of salivary glucose, salivary amylase, salivary total protein and salivary flow rate in diabetics in India. J Oral Sci 2010 Sep;52(3): 359-368.

6. Tagelsir A, Cauwels R, van Aken S, Vanobbergen J, Martens LC. Dental caries and dental care level (restorative index) in children with diabetes mellitus type 1. Int J Pediatr Dent 2011 Jan;21(1):13-22.

7. Prabhakar AR, Shubha AB, Mahantesh T. Estimation of calcium, phosphate and alpha amylase concentrations in stimulated whole saliva of children with different caries status: a comparative study. Malays Dent J 2008 Jan;29(1):6-13.

8. Moreira AR, Passos IA, Sampaio FC, Soares MS, Oliveira RJ. Flow rate, $\mathrm{pH}$ and calcium concentration of saliva of children and adolescents with type 1 diabetes mellitus. Braz J Med Biol Res 2009 Aug;42(8):707-711.

9. Abhikshyeet $P$, Ramesh V, Oza N. Glucose estimation in the salivary secretion of diabetes mellitus patients. Diabetes Metab Syndr Obes 2012 Jul;5:149-154.

10. Sreedevi, Shashikanth MC, Shambulingappa P. Comparison of serum glucose and salivary glucose in diabetic patients. J Indian Acad Oral Med Radiol 2008 Jan;20(1):9-13.

11. Belazi MA, Galli-Tsinopoulou A, Drakoulakos D, Fleva A, Papanayiotou PH. Salivary alterations in insulin-dependent diabetes mellitus. Int J Paediatr Dent 1998 Mar;8(1):29-33.

12. Bakianian Vaziri P, Vahedi M, Mortazavi H, Abdollahzadeh SH, Hajilooi M. Evaluation of salivary glucose, IgA and flow rate in diabetic patients: a case-control study. J Dent (Tehran) 2010 Winter;7(1):13-18.

13. Gheena S, Chandrasekhar T, Ramani P. Salivary characteristics of diabetic children. Braz J Oral Sci 2011 Apr-Jun;10(2): 93-97.

14. Shahbaz S, Katti G, Ghali SR, Katti C, Diwakar DD, Guduba V. Salivary alterations in type 1 diabetes mellitus patients: salivary glucose could be noninvasive tool for monitoring diabetes mellitus. Indian J Dent Res 2014 Jul-Aug;25(4): 420-424.

15. Kakoei S, Hosseini B, Haghdoost AA, Sanjari M, Hashemipour MA, Gholamhosseinian A. The detection of salivary glucose, caries and periodontal status in diabetes mellitus patients. J Oral Health Oral Epidemiol 2014 Jul;3(2):1-6.

16. Twetman S, Johansson I, Birkhed D, Nederfors T. Caries incidence in young type 1 diabetes mellitus patients in relation to metabolic control and caries-associated risk factors. Caries Res 2002 Jan-Feb;36(1):31-35.

17. Damle SG, Vidya I, Yadav R, Bhattal H, Loomba A. Quantitative determination of inorganic constituents in saliva and their relationship with dental caries experience in children. Dentistry 2012 Mar;2(3):1-5.

18. Lasisi TJ, Fasanmade AA. Comparative analysis of salivary glucose and electrolytes in diabetic individuals with periodontitis. Ann Ib Postgrad Med 2012 Jun;10(1):25-30.

19. Mulla JB. A study in between dental cavity and diabetes mellitus with reference to macro-minerals elements in Indian adults. Int J Basic Appl Med Sci 2013 Jan-Apr;3(1):170-175.

20. Bakas MJ. Analysis of effect of calcium, phosphorous \& alkaline phosphatase on dental caries in patients of diabetes. Int J Res Sci Technol 2015 Apr-Jun;5(2):18-25.

21. Karshan M. Do calcium and phosphorus in saliva differ significantly in caries-free and active-caries groups? Med J Dent Res 1941 Oct;15:83-86.

22. Hegde MN, Wali A, Punja A, Shetty C. Correlation between dental caries and alkaline phosphatase and calcium levels in serum and saliva in adult Indian population. Indo Am J Pharm Res 2014 Jan;4(4):2178-2182.

23. Jazaeri M, Malekzadeh H, Abdolsamadi H, Rezaei-Soufi L, Samami M. Relationship between salivary alkaline phosphatase enzyme activity and the concentrations of salivary calcium and phosphate ions. Cell J 2015 Spring;17(1):159-162.

24. Vijayaprasad KE, Ravichandra KS, Vasa AA, Suzan S. Relation of salivary calcium, phosphorus and alkaline phosphatase with the incidence of dental caries in children. J Indian Soc Pedod Prev Dent 2010 Jul-Sep;28(3):156-161.

25. Gandhy M, Damle SG. Relation of salivary inorganic phosphorus and alkaline phosphatase and the dental caries status in children. J Indian Soc Pedo Prev Dent 2003 Dec;21(4):135-138.

26. Rashkova M, Baleva M, Peneva M, Toneva N, Jegova G. Secretory immunoglobulin a ( $\operatorname{sg} A)$ and dental caries of children with different diseases and conditions influencing oral medium. J IMAB 2009;2:6-9.

27. Hegde AM, Naik N, Kumari S. Comparison of salivary calcium, phosphate and alkaline phosphatase levels in children with early childhood caries after administration of milk, cheese and GC tooth mousse: an in Vivo study. J Clin Pediatr Dent 2014 Summer;38(4):318-325.

28. Watanabe $Y$, Mizoguchi H, Masamura K, Naygaya T. The relationship of salivary flow rate and $\operatorname{sIgA}$ to dental caries in children. Environ Health Prev Med 1997 Oct;2(3): 122-125. 